\title{
Measurement of Accounting Information Accessibility and Understandability based on Distance of Information-state Transition Theory
}

\author{
Ping Guan, Jiayi Gu
}

School of Management, Shanghai University of Engineering Science, Shanghai 201620, China

\begin{abstract}
With the advent of the era of big data, the problem of information selection and decision efficiency becomes more and more important under the network environment. Accounting information is an important economic information resource, but it has been facing the dilemma between the usefulness and useable. Based on the theory of Distance of Information-state Transition, this paper describes several concepts related to information distance, discusses key issues such as target state information, state chain, transition probability, measurement rules and determine standards, analyzes the influence of information retrieval, social tagging, recommendation system and information navigation on information distance. Based on the measurement of accounting information acquisition and knowledge acquisition, this paper studies the optimization of information distance in the network environment, which provides theoretical support and practical reference for the realization of effective accounting information utilization and information architecture.
\end{abstract}

Keywords: Accounting Information; Accessibility; Distance of Information-state Transition; XBRL; Accounting Information Architecture.

\section{Introduction}

In the Age of Big Data, we no longer see the world as a series of events that we believe to be natural or social phenomena, but rather we realize that the world is essentially made up of information (Viktor, 2013). The essence of the world is information, and the essence of efficiency is deletion(Viktor, 2013). Information choice and data cleaning become increasingly important in a network environment. At present, the domestic use of the Internet and mobile Internet as a tool for information exchange and communication has become very popular. In order to improve the quality of accounting information, many scholars do research from the perspective of finance; only a few scholars do research from the point of view of information theory. In recent years, the revision of the International Financial Reporting Standards proposed that the financial report should be decomposed, and Standard \& Poor's international rating agency proposed that information disclosure should be stratified. Accounting information is an important channel of information and its enhancement cannot be separated from the development of information theory and information technology.

How does the Internet reporting system deal with information architecture? As far as the content, its qualitative characteristics are consistent including authenticity, faithful representation, comparability, verifiability and timely. Due the format is concerned, the design of the information lays more emphasis on its accessibility and understandability (Debreceny et al., 2002; Katherine, 2007; FASB, 2010).

The improvement of the quality of Internet financial report not only improves its content, but also improves its presentation. Its content should not only follow the disclosure requirements of financial reporting standards, but also fully consider the information needs of users. Its format should help to realize communication, design, interaction and pleasant experience, and realize the accessibility and understandability of financial reports with the aid of modern information technology.

How far is the distance between users and accounting information? In this paper, the author attempts to study the ways to improve the quality of accounting information in the network environment by employing the theory of Information-state Transition. Based on the theory of distance of Informationstate transition and a measurement model, this paper studies the influence of information retrieval, social tagging, recommendation systems, and information navigation on information distance. It is proposed that the deletion of unnecessary information state chains and the improvement of information 
state transition probabilities is key to reducing the information distance. Also, this paper studies the optimization of the information distance in a network environment.

\section{Distance of Information-state Transition Theory}

Quantitative information has been developed for nearly a century. In 1928, Hartley pointed out that measuring the amount of information contained in a message is by measuring freedom of choice, while the message itself contains nothing to the meaning and usefulness. In 1948, Shannon proposed the famous Shannon entropy calculations. Shannon thinks that the amount of information contained in a message is measured by reducing news of the uncertainty, Shannon also solve the problem of measurement of probability information. Later, many scholars are basically quantitative measurement of information along the idea of Hartley and Shannon's measure carried out. The concept of information distance from the professor Wang Huanchen in its "Information distance and information" (Wang Huanchen ,2006) writings were discussed in detail. Wang Huanchen defined as information is the characterization of the state of things, information distance can measure from probability of the state transition calculating by taking the logarithm.

In his theory, a quantitative measurement of information and knowledge, the convenience of information and knowledge acquisition, the convenience of management operation and the convenience of control operation are studied. By using probability theory and mathematical statistics, the theory of distance of Information-state transition provides a new theory and method to support the quantitative measurement of information accessibility and understandability.

\subsection{The Concepts}

The concepts and measurement models of The Distance of Information-state Transition theory as follows (Wang Huanchen, 2006):

(1) Information: The representation of the state.

(2) Information-state: refers to the state in which a thing can appear, such as signal state, text state, search state, operating state and so on.

(3) Information-state Transition: refers to the change of various possible states of a thing.

(4) Information state transition structure: refers to the structure of information state transfer chain, series or parallel structure.

(5) Distance of Information-state Transition: refers to a measure of the state transition process of a thing. Information distance can be measured from transition probability.

\subsection{Measurement Models}

The measurement of information distance mainly includes the information distance measure transferred between two states, the information distance measure transferred between multiple information states, and the information distance measure of combined form. The dimension unit of the information distance is dit, taking the logarithm of 2 as the low, and the model is as follows(Wang Huanchen, 2006):

Assume a transition from state $x_{i}$ to state $x_{j}$, the transition probability is $P_{i j}, i, j=1,2, \ldots, n$; The measure of the distance between two states is:

$$
\operatorname{DIT}(i j) \equiv d_{i j} \equiv \log \frac{1}{p_{i j}}=-\log p_{i j} ; \sum_{j=1}^{n} p_{i j}=1
$$

For the transition between multiple information states, it is measured by the transition probability matrix $\mathrm{P}$ and the information distance matrix $\mathrm{D}$ : 
Volume 13 (2021)

$$
D=-\log P \equiv-\left[\begin{array}{llll}
\log p_{11} & \log p_{12} & \mathrm{~L} & \log p_{1 n} \\
\log p_{21} & \log p_{22} & \mathrm{~L} & \log p_{2 n} \\
\mathrm{M} & \mathrm{M} & \mathrm{M} & \\
\log p_{n 1} & \log p_{n 2} & \mathrm{~L} & \log p_{n n}
\end{array}\right]=\left[\begin{array}{cccc}
d_{11} & d_{12} & \mathrm{~L} & d_{1 n} \\
d_{21} & d_{22} & \mathrm{~L} & d_{2 n} \\
\mathrm{M} & \mathrm{M} & \mathrm{M} \\
d_{n 1} & d_{n 2} & \mathrm{~L} & d_{n n}
\end{array}\right]
$$

When a continuous state transition of information is encountered, a combined state transition structure is generated. This combination of information has two basic forms, namely series connection and parallel connection, and the measurement formula is:

$$
\begin{array}{r}
\operatorname{DIT}\left(i_{1}, j_{M}\right)=\sum_{m=1}^{M} \operatorname{DIT}\left(i_{m}, j_{m}\right) ; \quad p\left(i_{1}, j_{M}\right)=\prod_{m=1}^{M} p\left(i_{m}, j_{m}\right) \\
\operatorname{DIT}(i, j)=\sum_{m=1}^{M} D I T\left(i_{m}, j_{m}\right) ; \quad p(i, j)=\prod_{m=1}^{M} p\left(i_{m}, j_{m}\right)
\end{array}
$$

The figure of serial form information structure and parallel form information structure are as follow Figure 1 and Figure 2:

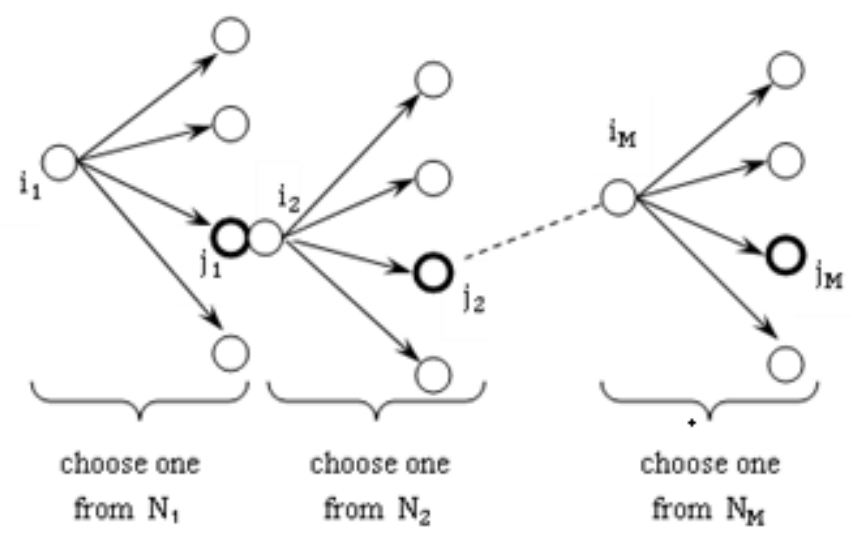

Figure 1. Schematic diagram of the serial form information structure

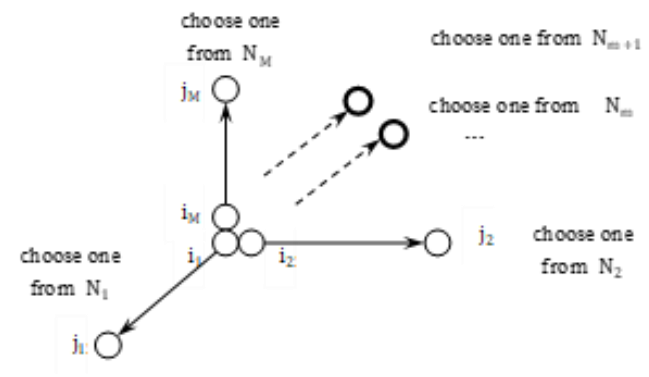

Figure 2. Schematic diagram of parallel form information structure

\subsection{Several Key Issues}

\section{1) Target status}

There is a common requirement in the operation of information, that is target state. In the course of effective measurement, target state exists and can be realized. "state chain breaking" and " false information" are special cases in which the target state cannot be achieved, and the measurement results are infinite. In addition, the realization path of the target state can be either one path or multiple paths. If the target information status is "broken" or "missing", the result of the information distance is infinite and it is invalid. 


\section{2) Transition Probability}

Transfer probability is an important concept in Markov chain. Information distance is the quantitative measurement from "initial state" to "target state". Information state and transition probability are two key factors in measuring information distance. As we know, Quantification of information is measured by probability, while the distance of Information-state transition is measured by transition probability.

\subsection{Measure Rules}

In practice, we must also set some rules to ensure the calculation of effective information distance. Usually, it is assumed state selection and network operation is considered. Information distance of state selection is determined by times and probability of state transition. The more the number of state transitions, the longer the information state chain, the greater the information distance; The information state transition probability is closer to 1 , the information distance is closer to 0 ; on the contrary, the information state transition probability is closer to 0 , the information distance closer to infinity (see Table 1). Such as bookmarks, use bookmarks so that we can quickly find the reading place last time to read.

Table 1. Information Distance Measuring

\begin{tabular}{|c|c|c|}
\hline $\begin{array}{c}\text { The information state transition } \\
\text { description }\end{array}$ & $\begin{array}{c}\text { The information state transition } \\
\text { probability }\end{array}$ & Information distance (dit) \\
\hline Immediate action & 1 & 0 \\
\hline Some experience & $1 / 2<\mathrm{P}<1$ & $0<\mathrm{DIT}<1$ \\
\hline equal & 0.5 & 1 \\
\hline No experience & $1 / 2$ & 1 \\
\hline Don't know how to operate & $0<\mathrm{P}<1 / 2$ & DIT $>1$ \\
\hline
\end{tabular}

The information distance of network operation is decided of keys, clicks, inputs and scans. It is affected by hardware and software environment, user operation level and so on. Usually, only the number of operations is measured. The following are the rule setting for the above two main aspects: (1)Information status selection, in order to reach the target status (choose one from $\mathrm{N}$ ), such as menu items, information modules, etc. (2) Press the button, click or not(choose one from two), such as keyboard click, mouse click, etc. In this way, the calculation formula of the information distance can be expressed as: DIT $(\mathrm{I}, \mathrm{J})=\langle\mathrm{p}(\mathrm{i} 1, \mathrm{im})>+[\mathrm{m}]$.

\subsection{The Influence of Network Information Technology on Information Distance}

In the network environment, search engines make it easier for us to get useful information and reduce the problem of information asymmetry; tags and navigation make our path to the target state more optimized and reduce the problem of detours ( Morville et al., 2006;Vicky et al., 2012); Information retrieval, information label and information navigation can reduce the information distance. Its essence is to delete the unnecessary information state chain, or delete the unnecessary information state transition direction, which improves the probability of information state transition, thus shortening the information distance(GuanPing et al., 2017). At the same time, the introduction of recommendation in retrieval system, label system and navigation system can help information users to clarify their goals and translate potential information needs into final actual needs. Recommendation systems make the target information state more explicit and reduce the problem of information demand fuzziness. Redundant information, false information, multiple selection and cumbersome operation constitute the increment of information distance, while information classification, information ranking, information identification and resource recommendation are the subtractions of information distance. 


\section{Evalution}

\subsection{Internet Access to Information and Business Transaction}

Example 1: Information distance measurement of Internet financial report

Internet access to information

Internet financial report is the most important component of Internet Investor Relations( Bollen et al., 2006;2008). eXtensible Business Reporting Language(XBRL) is a markup language which is based on XML.XBRL is the latest technology for unstructured information processing, especially the financial information processing. XBRL can accurately marks the financial statements and thousands of data contained in the notes, making it easy for information users to access and analyze data(Janvrin et al., 2013). It represents the direction of the future network financial reporting.

(1) target state: access the information from annual report

(2) state chain and transition probability: choose one from $\mathrm{N}$

The general path for PDF report data acquisition is: The official website of the stock exchange $\rightarrow$ select the information disclosure in the menu item(choose one from seven) $\rightarrow$ click $\rightarrow$ select the Listed company information(choose one from eight) $\rightarrow$ click $\rightarrow$ select periodic report(choose one from eight) $\rightarrow$ click $\rightarrow$ enter stock code, query year, report type, Plate type $\rightarrow$ click query $\rightarrow$ click annual report $\rightarrow$ select target information(such as two hundreds, choose one from two hundreds) $\rightarrow$ click.

The general path for XBRL report data acquisition is: Stock Exchange official website $\rightarrow$ select the information disclosure in the menu item(choose one from seven) $\rightarrow$ click $\rightarrow$ select the listed company information in the menu item(choose one from eight) $\rightarrow$ click $\rightarrow$ select the XBRL instance document in the menu item ( choose one from eight) $\rightarrow$ click $\rightarrow$ enter the stock code, report type $\rightarrow$ click $\rightarrow$ select the target information(choose one from six) $\rightarrow$ click.

Information distance measure of two reporting modes as follows Table 2.

Table 2. Information Distance Measure of Two Reporting Modes (a)

\begin{tabular}{|c|c|c|c|c|}
\hline Reporting mode & Calculation & State selection & Click & DIT \\
\hline PDF & $\langle 1 / 7>+<1 / 8>+<1 / 8>+<1 / 200>+6[1]$ & 16.45 & 6 & 22.45 \\
\hline XBRL & $\langle 1 / 7>+<1 / 8>+<1 / 8>+<1 / 6>+5[1]$ & 11.39 & 5 & 16.39 \\
\hline
\end{tabular}

Further, when investors need to compare the corresponding data: Information distance measure of two reporting modes as follows Table 3.

Table 3. Information Distance Measure of Two Reporting Modes (b)

\begin{tabular}{|c|c|c|c|c|}
\hline Reporting mode & Calculation & State selection & click & DIT \\
\hline PDF & $\langle 1 / 7>+\langle 1 / 8>+<1 / 8>+<1 / 200\rangle+<1 / 200>+9[1]$ & 24.10 & 9 & 33.10 \\
\hline XBRL & $\langle 1 / 7>+<1 / 8>+<1 / 8>+<1 / 6>+5[1]$ & 11.39 & 5 & 16.39 \\
\hline
\end{tabular}

From the calculation, we can see that XBRL provides much convenience for investors to filter, compare and combine data, greatly reducing the information distance during information utilization. When the content of financial report is authentic, public investors especially small and medium-sized investors experience a certain degree of difficulty in the acquisition of information and its analysis. Meanwhile, information asymmetry, misleading information and false information make the farthest information distance for the information users. As a result, decision-making becomes difficult.

Example 2: Information distance measurement of Online accounting books purchasing

Internet business transaction

Taking business transaction as an example to measure and compare the information distance between Amazon (Website) and Amazon (App), Mobile Application App is a software system installed and running on a mobile communication device, which is a window for mobile communication users to access the Internet and obtain network services. With the rapid development 
of the mobile internet, more and more users hope to quickly conduct online business transactions through an App installed on a smart mobile device.

(1) Target state: purchase the latest Accounting book with the highest user rating on Amazon.

(2) State chain and transition probability: choose one from N.

The general path for website purchases is : Amazon Homepage $\rightarrow$ Input Accounting and finance in the search box $\rightarrow$ click $\rightarrow$ click sort $\rightarrow$ select shelf time(choose one from five) $\rightarrow$ click $\rightarrow$ select customer ratings (choose one from five) $\rightarrow$ click $\rightarrow$ click $\rightarrow$ select "buy now" or "place one order" (choose one from two) $\rightarrow$ click $\rightarrow$ Enter the account $\rightarrow$ click continue $\rightarrow$ enter password $\rightarrow$ click login $\rightarrow$ select payment method (choose one from three) $\rightarrow$ click Alipay $\rightarrow$ click continue $\rightarrow$ scan payment code $\rightarrow$ enter payment password $\rightarrow$ payment is completed.

The general path of APP purchases is: Amazon APP Homepage $\rightarrow$ Enter Accounting and finance in the search box $\rightarrow$ click filter $\rightarrow$ click sort $\rightarrow$ select shelf time (choose one from five) $\rightarrow$ click $\rightarrow$ click filter $\rightarrow$ click filter $\rightarrow$ select customer ratings (choose one from five) $\rightarrow$ click $\rightarrow$ select "buy now" or "place one order" (choose one from two) $\rightarrow$ click $\rightarrow$ enter Account, Password $\rightarrow$ click login $\rightarrow$ select payment method (choose one from two) $\rightarrow$ click login Alipay $\rightarrow$ click $\rightarrow$ enter Payment Password $\rightarrow$ Payment complete.

(1) Measure rule: consider the information distance of the state selection and click operation, and ignore the keyboard and input operations.

(2) Information distance measure of two ways as follows Table 4.

Table 4. Information Distance Measure of Two Ways

\begin{tabular}{|c|c|c|c|c|}
\hline \multirow{2}{*}{ Reporting mode } & \multirow{2}{*}{ Calculation } & \multicolumn{3}{|c|}{ results } \\
\cline { 3 - 5 } & & State selection & click & DIT \\
\hline Website & $\langle 1 / 5\rangle+\langle 1 / 5\rangle+\langle 1 / 2\rangle+\langle 1 / 3\rangle+10[1]$ & 7.23 & 10 & 17.23 \\
\hline App & $\langle 1 / 5\rangle+\langle 1 / 5\rangle+\langle 1 / 2\rangle+\langle 1 / 2\rangle+10[1]$ & 6.64 & 10 & 16.64 \\
\hline
\end{tabular}

From the measurement results and comparison, APP has seized several key links in the online shopping process, making it easier for mobile phone customers to purchase than for website purchases. This is one of the most important factors for the rapid development of mobile Internet in recent years.

\subsection{Information Distance Measurement of Accounting Research Literature Acquisition}

Taking ScienceDirect as an example, the general search in the foreign language database includes Keywords, Author name, Journal/Book title, Volume, Issue and Page. In the advanced search, the search elements are expended into All fields, Abstract/Title/Keywords, Authors, Specific Author, Source Title, Title, Keywords, Abstract, References, ISSN, ISBN and Affiliation, total of twelve search elements are set, and Year, Publication Title, Topic, and Content type four controls are set. This network knowledge acquisition process is a multi-continuous information state transition, which is independent of each other, regardless of the order, and needs to be considered at the same time. It is a parallel state information state transition structure. The total information distance is the weighted sum of the distances of the local information. The specific path of knowledge retrieval is shown in the Table 5 .

Table 5. Get the path of a foreign database document

\begin{tabular}{|c|cc|cc|}
\hline Search & Search element & \multicolumn{2}{c|}{ Control item } \\
\hline Search element & $1 / 12$ & $1 / 12$ & $1 / 12$ & $1 / 4$ \\
\hline Control item & $1 / 4$ & $1 / 12$ & $1 / 4$ & $1 / 4$ \\
\hline
\end{tabular}

The following calculations are performed to input and select two search elements; one search element and one control item; two search elements and one control item; one search element and two control items; two search elements and two control items. Number of documents of different technologies in different situations as follows Table 6. 
Table 6. Number of documents of three accounting information technologies in different situations

\begin{tabular}{|c|c|c|c|c|}
\hline \multirow{2}{*}{ Element and control number } & \multirow{2}{*}{ Element and control } & \multicolumn{3}{|c|}{ Number of documents } \\
\cline { 3 - 5 } & & Big data & Block chain & XBRL \\
\hline Two elements & Keywords; title & 2612 & 220 & 44 \\
\hline One element One control & Keywords; Year & 2088 & 539 & 4 \\
\hline Two elements One control & Keywords ;title; Year & 631 & 9 & 2 \\
\hline One element Two controls & Keywords Year; Content type & 411 & 145 & 3 \\
\hline Two elements Two controls & Keywords; title Year ;Content type & 129 & 3 & 2 \\
\hline
\end{tabular}

(Year: 2018; Content type: Journal)

Information distance measure of three accounting information technologies as follows Table 7.

Table 7. Information distance measure of three accounting information technologies

\begin{tabular}{|c|c|c|}
\hline $\begin{array}{l}\text { Technology } \\
\text { name }\end{array}$ & Calculation & DIT \\
\hline \multirow{5}{*}{ Big data } & $\mathrm{d} 1=\log 12+\log 2+\log 12+\log 2+\log 4+\log 2+\log 4+\log 2+\log 129+\log 2=23.18 \mathrm{dit}$ & \multirow{5}{*}{ 21.4dit } \\
\hline & $\mathrm{d} 2=\log 12+\log 2+\log 12+\log 2+\log 4+\log 2+\log 631+\log 2=22.47 \mathrm{dit}$ & \\
\hline & $\mathrm{d} 3=\log 12+\log 2+\log 12+\log 2+\log 2612+\log 2=21.52 \mathrm{dit}$ & \\
\hline & $\mathrm{d} 4=\log 12+\log 2+\log 4+\operatorname{lo} 2+\log 4+\log 2+\log 411+\log 2=20.27 \mathrm{dit}$ & \\
\hline & $\mathrm{d} 5=\log 12+\log 2+\log 4+\log 2+\log 2088+\log 2=19.61 \mathrm{dit}$ & \\
\hline \multirow{5}{*}{ Block chain } & $\mathrm{d} 1=\log 12+\log 2+\log 4+\operatorname{lo} 2+\log 4+\log 2+\log 145+\log 2=18.76 \mathrm{dit}$ & \multirow{5}{*}{$17.7 \mathrm{dit}$} \\
\hline & $\mathrm{d} 2=\log 12+\log 2+\log 12+\log 2+\log 220+\log 2=17.95 \mathrm{dit}$ & \\
\hline & $\mathrm{d} 3=\log 12+\log 2+\log 12+\log 2+\log 4+\log 2+\log 4+\log 2+\log 3+\log 2=17.75 \mathrm{dit}$ & \\
\hline & $\mathrm{d} 4=\log 12+\log 2+\log 4+\log 2+\log 539+\log 2=17.66 \mathrm{dit}$ & \\
\hline & $\mathrm{d} 5=\log 12+\log 2+\log 12+\log 2+\log 4+\log 2+\log 9+\log 2=16.34 \mathrm{dit}$ & \\
\hline \multirow{5}{*}{ XBRL } & $\mathrm{d} 1=\log 12+\log 2+\log 12+\log 2+\log 4+\log 2+\log 4+\log 2+\log 2+\log 2=17.17 \mathrm{dit}$ & \multirow{5}{*}{$14.1 \mathrm{dit}$} \\
\hline & $\mathrm{d} 2=\log 12+\log 2+\log 12+\log 2+\log 44+\log 2=15.63 \mathrm{dit}$ & \\
\hline & $\mathrm{d} 3=\log 12+\log 2+\log 12+\log 2+\log 4+\log 2+\log 2+\log 2=14.17 \mathrm{dit}$ & \\
\hline & $\mathrm{d} 4=\log 12+\log 2+\log 4+\operatorname{lo} 2+\log 4+\log 2+\log 3+\log 2=13.17 \mathrm{dit}$ & \\
\hline & $\mathrm{d} 5=\log 12+\log 2+\log 4+\log 2+\log 4+\log 2=10.58 \mathrm{dit}$ & \\
\hline
\end{tabular}

(Comprehensive information distance calculation under equal weights: $d=\sum_{i=1}^{5}$ widi)

Through the measurement, it is found that different search elements and control items are input, the obtained target results are different, and the information distance is not equal. When the number of documents in the search results is large, the information distance is very large.

\section{Conclusion}

The quality characteristics of financial report and those of information resources are the same in essence, both paying attention to authenticity, relevance, comparability, verifiability and timely in the content; but concerning its format, financial report put more emphasis on accessibility and understandability.

From the perspective of information providers and managers, they demand more on the usability of information; while from the perspective of users, they emphasize accessibility. Based on the theory of Distance of Information-state Transition and measurement model, the empirical research results show that the information distance between users and accounting information is caused by the following elements: Due to highly specialized accounting terms, ineffective analysis tools and information overload, which increase the difficulty of selection and utilization.

\section{Acknowledgments}

This paper is supported by audit theory and practice course construction project. 


\section{References}

[1] Viktor Mayer-Schönberger. 2013.Big Data: A Revolution That Will Transform How We Live, Work, and Think.

[2] Viktor Mayer-Schönberger.2013. Delete: The Virtue of Forgetting in the Digital Age.

[3] Victoria Chiu, Qi Liu, Brigitte Muehlmann, Amelia Annette Baldwin, 2019. A bibliometric analysis of accounting information systems journals and their emerging technologies contributions, Int. J. Account. Inf. Syst. (32): 24-43.

[4] IASB, 2010. Conceptual Framework for Financial Reporting: Chapter 1, the Objective of GeneralPurpose Financial Reporting.

[5] FASB, 2010. SFAC No.8: Chapter 1, the Objective of General-Purpose Financial Reporting, Chapter 3, Qualitative Characteristic of Useful Financial Information, (9).

[6] Katherine Schipper, 2007. Required Disclosures in Financial Reports, the Accounting Review, 82(2):301326.

[7] Vicky Arnold, Jean C. Bedard, Jillian R. Phillips, Steve G. Sutton, 2012.The impact of tagging qualitative financial information on investor decision making: Implications for XBRL, Int. J. Account. Inf. Syst. (13): $2-20$.

[8] Wang Huanchen, 2006. Information Distance and Information, Beijing: Science Press.

[9] Morville P, Rosenfeld L., 2006.Information Architecture for the World Wide Web: Designing LargeScale Web Site [M].3rd Revised edition. O'Reilly Media, Inc.

[10] Wang Huanchen.2011. System information metric. Journal of University of Shanghai for Science and Technology 33(6): 631-640, 508.

[11] GuanPing, Song LiangRong., 2017.Measurement and Optimization of Information Distance in Network Environment. Journal of the China Society for Scientific and Technical Information,36(12):1302-1308.

[12] Bollen L H, Hassink H F, Bozic G. 2006. Measuring and Explaining the Quality of Internet Investor Relations Activities: A Multinational Empirical Analysis Int. J. Account. Inf. Syst. 7(4): 273-298.

[13] Seán O'Riain, Edward Curry, Andreas Harth, 2019.XBRL and open data for global financial ecosystems: A linked data approach, Int. J. Account. Inf. Syst. (13):141-162.

[14] Janvrin D J, Pinsker R E, Mascha M F. 2013.XBRL-Enabled, Spreadsheet, or PDF? Factors Influencing Exclusive User Choice of Reporting Technology. Journal of information system. (27) 2:35-49.

[15] Andrew J. Felo, Joung W. Kim, Jee-Hae Lim, 2018.Can XBRL detailed tagging of footnotes improve financial analysts' information environment? Int. J. Account. Inf. Syst. (28):45-58.

[16] Debreceny R, Gray G L, Rahman A. 2002.The Determinants of Internet Financial Reporting. Journal of Accounting and Public Policy,21(4-5):371-394.

[17] Bollen L H, Hassink H F, Lange R K, Buijl S D. 2008. Best practices in managing investor relations websites: directions for future research. J. Inf. Syst. 22(2):171-194.

[18] Esra'a Alkhatib, Hannu Ojala, Jill Collis, 2019. Determinants of the voluntary adoption of digital reporting by small private companies to Companies House: Evidence from the UK, Int. J. Account. Inf. Syst. (34): 100421.

[19] John McCallig, Alastair Robb, Fiona Rohde, 2019. Establishing the representational faithfulness of financial accounting information using multiparty security, network analysis and a blockchain, Int. J. Account. Inf. Syst. (33):47-58. 